\title{
AN SMDP-BASED INITIAL RADIO ACCESS TECHNOLOGY SELECTION METHOD FOR HETEROGENEOUS WIRELESS NETWORKS
}

\author{
by \\ Kazi Reazul Islam \\ M.Eng., ECE, Ryerson University, Toronto, Canada, 2010
}

\author{
A thesis \\ presented to Ryerson University \\ in partial fulfilment of the \\ requirements for the degree of \\ Master of Science \\ in the Program of \\ Computer Science \\ Toronto, Ontario, Canada, 2014 \\ (C)Kazi Reazul Islam 2014
}




\section{AUTHOR'S DECLARATION FOR ELECTRONIC SUBMISSION OF A THESIS}

I hereby declare that I am the sole author of this thesis. This is a true copy of the thesis, including the required final revisions, as accepted by my examiners.

I authorize Ryerson University to lend this thesis to other institutions or individuals for the purpose of scholarly research.

I further authorize Ryerson University to reproduce this thesis by photocopying or by other means, in total or in part, at the request of other institutions or individuals for the purpose of scholarly research.

I understand that my thesis may be made electronically available to the public.

Kazi Reazul Islam 


\title{
AN SMDP-BASED INITIAL RADIO ACCESS TECHNOLOGY SELECTION METHOD FOR HETEROGENEOUS WIRELESS NETWORKS
}

\author{
Kazi Reazul Islam \\ MSc, Computer Science, Ryerson University, 2014
}

\begin{abstract}
Heterogeneous Wireless Networks (HetNets) are a class of networks that have been designed to provide an open and flexible architecture for the coexistence of various distinct Radio Access Technologies (RATs). In such networks, one of the key challenges is the selection of the best available RATs when a user is connected upon making an incoming service request. This thesis proposes a Joint Call Admission Control (JCAC) - based approach for initial RAT selection in HetNets composed of two co-located wireless networks . The RAT selection problem in such HetNet is formulated by using a JCAC-based Semi-Markov Decision Process (SMDP) model, where the JCAC policy is determined according to a network cost function that weights three parameters: a cost of blocking the incoming service request, a cost of accessing RAT, and the RAT energy consumption cost. Simulation results are provided, showing the benefits of our approach in terms of RAT selection and resulting network energy consumption cost .
\end{abstract}




\section{Acknowledgments}

I like to give my gratefulness to my supervisor, Dr. Isaac Woungang for accepting me as a Masters student and for supervising my work with patience. Without his continual support and thoughtful mentoring, this thesis would not be possible.

Moreover, my appreciation goes to my friends in our lab who were there when I needed help.

I dedicate this thesis to my family: my wife, my son, my daughter and my parents who supported me unconditionally and have been the source of motivation and inspiration for me and made my time more enjoyable. 
To my family for their kindness and encouragement. 


\section{Contents}

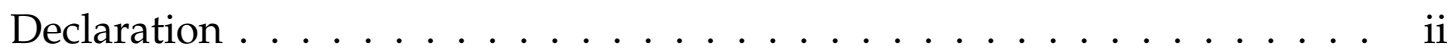

Abstract. ................................... iii

Acknowledgments . . . . . . . . . . . . . . . iv

Dedication ......................

Table of Contents . . . . . . . . . . . . . . . . . vii

List of Figures $\ldots \ldots \ldots \ldots \ldots$. . . . . . . . . . . . . . . . . . . . . . . .

List of Tables . . . . . . . . . . . . . . . . . . . . ix

List of Abbreviations . . . . . . . . . . . . . . . $x$

$\begin{array}{lll}1 & \text { Introduction } & 1\end{array}$

1.1 Motivation . . . . . . . . . . . . . . . . . 1

1.2 Problem Statement . . . . . . . . . . . . . . . . . . . . . 3

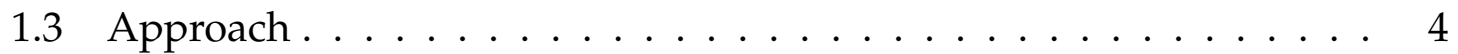

1.4 Thesis Contributions $\ldots \ldots \ldots \ldots \ldots \ldots \ldots$

1.5 Thesis Organization $\ldots \ldots \ldots \ldots \ldots \ldots$

\begin{tabular}{|lll}
2 & Background and Literature Review & 7
\end{tabular}

2.1 Heterogeneous Wireless Networks $\ldots \ldots \ldots \ldots \ldots$. . . . . . . 7

$2.2 \quad$ Radio Resource Management in Heterogeneous Wireless Networks . 9

2.3 Literature Review . . . . . . . . . . . . . . . . . . . 12

$\begin{array}{lll}3 & \text { Methodology } & 16\end{array}$

3.1 System Model . . . . . . . . . . . . . . . . . . . . . . . . . . 16

3.2 Problem Formulation . . . . . . . . . . . . . . . . . . . . 17

3.2 .1 SMDP Model . . . . . . . . . . . . . . . . . . . . 17

3.2 .2 State Spaces . . . . . . . . . . . . . . . . . . . . . . . 17

3.2.3 Decision Epochs With Actions $\ldots \ldots \ldots$

3.2.4 $\quad$ Expected Time Until The Next Decision Epoch . . . . . . . . . 18

3.2 .5 Transition Probabilities $\ldots \ldots \ldots \ldots$

3.2 .6 Cost Function . . . . . . . . . . . . . . . . . . 20

3.2 .7 JCAC Policy . . . . . . . . . . . . . . . . . . 21 
\begin{tabular}{|llll}
4 & Performance Evaluation & 24
\end{tabular}

4.1 Simulation Tool and Parameters. . . . . . . . . . . . . . . . . . 24

4.2 Performance Measurement . . . . . . . . . . . . . . . . . . . 25

4.3 Simulation Results . . . . . . . . . . . . . . . . . . . . . . . 26

4.4 Simulation Results $\ldots \ldots \ldots \ldots$

4.4 .1 Results for Scenario - I . . . . . . . . . . . . . . . 28

4.4 .2 Results for Scenario - II . . . . . . . . . . . . . . . . 30

4.4 .3 Results for Scenario III . . . . . . . . . . . . . . . . . . 32

4.4 .4 Results for Scenario IV . . . . . . . . . . . . . . . . . . 35

$4.5 \quad$ Analysis of the Optimal Structure of Initial RAT Selection Policy . . . 38 4.5.1 Analysis of Class-1 Call Accepted by the Optimal JCAC: . . . 39

5 Conclusion 42

\begin{tabular}{ll}
\hline Bibliography & 45
\end{tabular} 


\section{List of Figures}

1.1 A HetNet Roaming Scenario . . . . . . . . . . . . . . . . . . . . 2

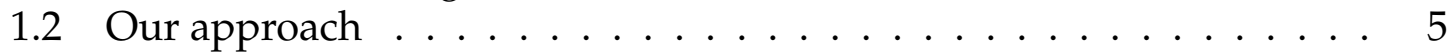

4.1 Scenario I - Blocking probability vs. Traffic intensities $\mid$ (a) $\mid$ Case of class-1 calls and $($ b) $\mid$ Case of class-2 calls. . . . . . . . . . . . 28

4.2 Scenario I - RAT utilization vs. Traffic intensities (a) $\mid$ Case of RAT1 and (b)|Case of RAT2. . . . . . . . . . . . . . . . . . . . . . . . . . . . . . . 29

4.3 Scenario I - Optimal cost vs. Traffic intensities. . . . . . . . . . . . . 30

\begin{tabular}{|ll|l|}
\hline 4.4 & Scenario II - Blocking probability vs. Bandwidth|(a)|Case of class-1 \\
\hline
\end{tabular} call and $\mid(b)$ Case of class-2 call. $\ldots \ldots \ldots \ldots \ldots$. . . . . . . . . 30

$4.5 \quad$ Scenario II - RAT utilization vs. Bandwidth $\mid$ (a) $\mid$ Case of RAT1 and $|(\mathrm{b})|$ Case of RAT2 $\ldots \ldots \ldots \ldots \ldots \ldots \ldots \ldots \ldots$

$4.6 \quad$ Scenario II - Optimal cost vs. Bandwidth. . . . . . . . . . . . . . 32

4.7 Scenario III - Blocking probability vs. Weight of energy consumption cost in total cost (a) Case of class-1 calls and(b)|Case of class-2 calls.) . 33

$4.8 \quad$ Scenario III - RAT utilization vs. Weight of energy consumption cost in total cost (a) Case of RAT1 and (b)|Case of RAT2. $\ldots \ldots$. . . . . 34

$4.9 \quad$ Scenario III - Optimal cost vs. Weight for energy consumption cost

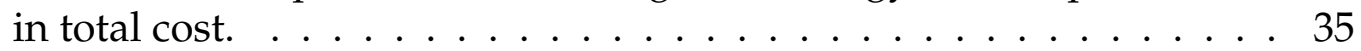

4.10 Scenario IV - Blocking probability vs. Channel holding time . . . . . 36

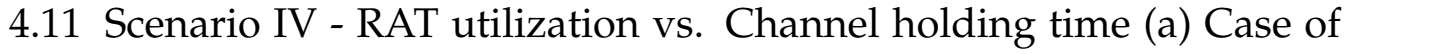
RAT1 and $(\mathrm{b}) \mid$ Case of RAT2 $\ldots \ldots \ldots \ldots \ldots \ldots$

4.12 Scenario IV - Optimal cost vs. Channel holding time. . . . . . . . . 38 


\section{List of Tables}

$4.1 \quad$ Fixed parameters values for simulation $\ldots \ldots \ldots \ldots \ldots \ldots$

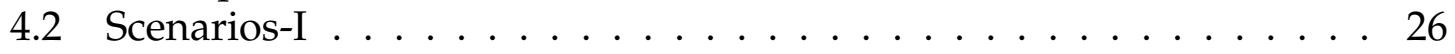

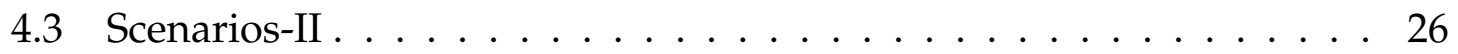

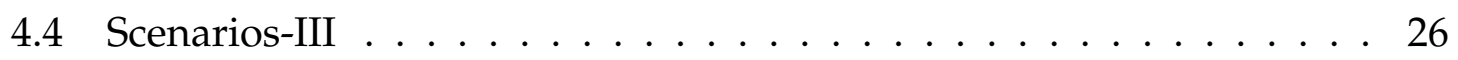

4.5 Scenarios-IV . . . . . . . . . . . . . . . . . . . . . . . . 26

4.6 Optimal Policy for the System Configuration . . . . . . . . . . . . 39

4.7 When RAT1 channel load is less than $12 \ldots \ldots$. . . . . . . . . 40

4.8 When RAT1 channel load is less than $16 \ldots \ldots$. . . . . . . . . . 41

4.9 When RAT1 channel load is Grater than $22 \ldots \ldots$ 


\section{List of Abbreviations}

- 3GPP: Third Generation Partnership Project

- B3G: Beyond 3G

- 4G: Fourth Generation

- RRM: Radio Resource Management

- CRRM: Common RRM

- JRRM: Joint Radio Resource Management

- JCAC: Joint Call Admission Control

- CAC: Call Admission Control

- AHP: Analytical Hierarchy Process

- AP: Access Point

- CDMA: Code Division Multiple Access

- CRRM: Common Radio Resource Management

- FDD: Frequency-division Duplexing 
- FDMA: Frequency Division Multiple Access

- GPRS: General packet radio service

- GSM: Global System for Mobile Communication

- HetNets: Heterogeneous Wireless Networks

- MDP: Markov Decision Process

- OFDM: Orthogonal frequency-division multiplexing

- QoS: Quality of Service

- RAT: Radio Access Technology

- SAE: System Architecture Evolution

- SMDP: Semi-Markov Decision Process

- TDD: Time-division Duplexing

- TDMA: Time Division Multiple Access

- UE: User Equipment

- UMTS: Universal Mobile Telecommunications System

- WiMax: Worldwide Interoperability for Microwave Access

- WLAN: Wireless Local Area Network 


\section{Chapter 1}

\section{Introduction}

\subsection{Motivation}

HetNets (Fig. 1.1) have been proposed to provide an open and flexible architecture for the coexistence of a wide variety of Radio Access Technologies (RATs). These RATs have diverse features for each network in terms of coverage, implementation and operational cost [1]. They also offer different features such as transmission rates, coverage, and cost / revenue, which make it possible to increase the overall performance compared to the performances that can be offered by stand-alone networks [2]. Radio Resource Management (RRM) techniques can be used to ensure an effective and coordinated use of the available radio resources in HetNets. From an operational viewpoint, HetNets should follow the System Architecture Evolution (SAE) standards [3]. In SAE, the functionality of the network determines the choice of the RAT. This choice is divided in two unique phases: network discovery, and User Equipment (UE), which help determining the available 


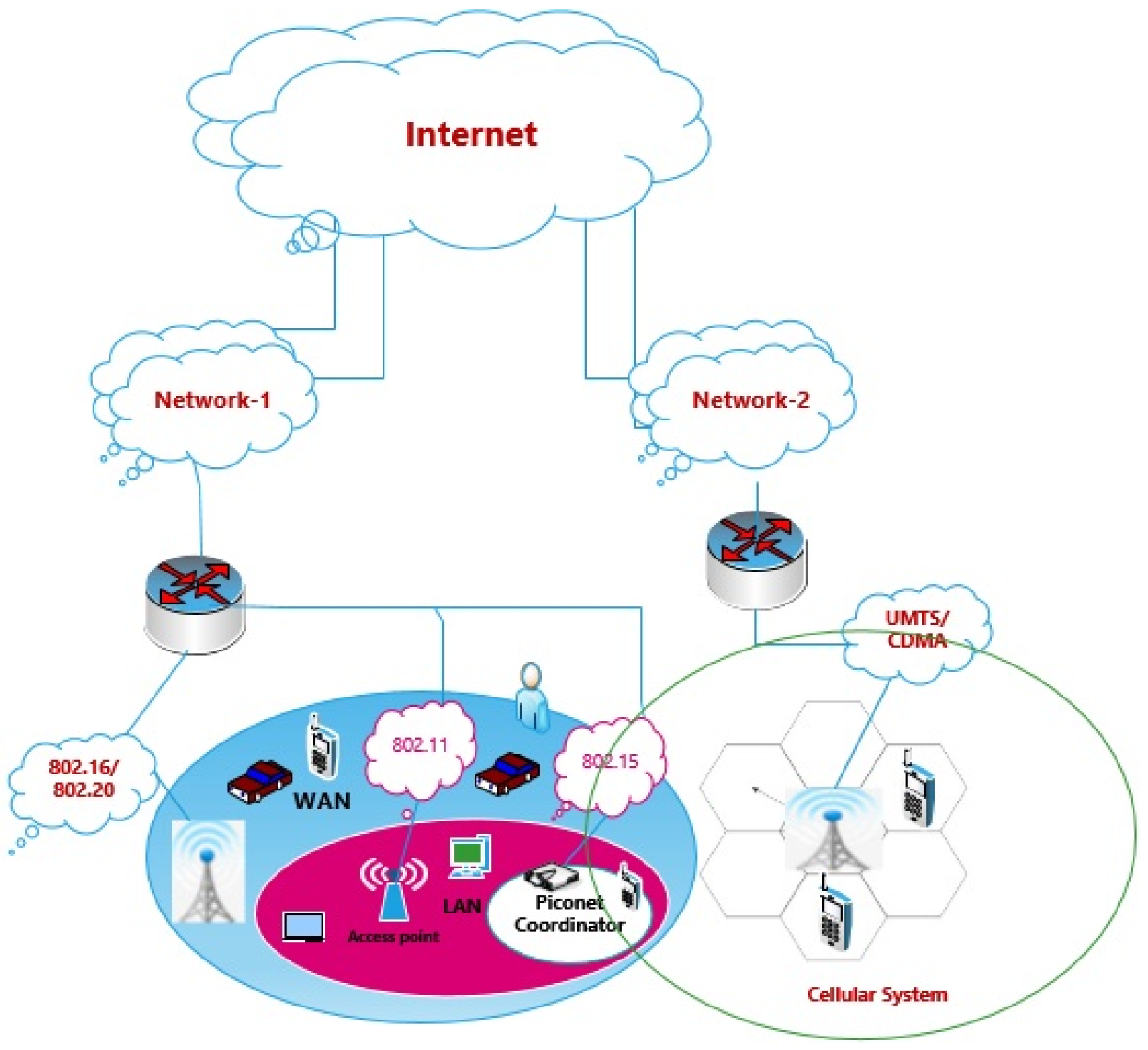

Figure 1.1: A HetNet Roaming Scenario

RATs in an efficient way. For this reason, the availability of RATs must be given by the network according to the target UE based on their locations. Depending on this information, a UE could select the best RATs to be connected to. In order to achieve this, it uses some factors such as energy consumption, traffic load at each RAT etc, to make a decision. 


\subsection{Problem Statement}

This thesis promotes the idea that rather than focusing the decision of network discovery and RAT selection in the hand of an User Equipment (UE), it would be more appropriate to have an external agent to perform this decision. We believe that this role can be played by a Radio Resource Management (RRM) algorithm that will not only provide a global coverage of the network, but will also support different applications and services, from the various RATs present in the HetNets environment. Following this trend, the Common RRM (CRRM) [2] and the Joint RRM (JRRM) [4] are the two benchmark RRM frameworks that have been proposed for HetNets. The RAT selection procedure is one of the most important functions within the CRRM concept, which determines whether an incoming service request should be accepted or rejected. It also determines which of the available RATs is best suited to accommodate the incoming service request, so that the user's information can be transmitted.

On the other hand, the Joint Call Admission Control (JCAC) scheme [5] in JRRM plays a central role in a HetNet environment since it defines how the radio resources or wireless channels can be efficiently shared among the incoming service requests. The JCAC operation involves determining whether an incoming service request is accepted or not according to an admission constraint. If that constraint is satisfied, the request will be accepted; otherwise, it will be blocked. The JCAC operation also selects in which of the available RATs an incoming service request will be accommodated [6]. 


\subsection{Approach}

The problem of RAT selection in HetNets is formulated as a JCAC-based SemiMarkov Decision Process (SMDP) model, where the decision of selecting the best RAT among available ones is determined by means of a cost function that weights three criteria: a cost associated with the blocking of a user incoming service request - this takes into account the priority assigned to each service class in each RAT; an energy consumption cost - which measures the overall network battery power savings; and the network access cost. More precisely, upon an incoming service request from the user, based on three criteria which are blocking probability, access price, and energy consumption, the JCAC-based SMDP scheme determines the suitable RAT to handle the request. This decision is made according to the resulting optimal JCAC policy, derived by converting the continuous time SMDP model into

a discrete time MDP model and by using the Value Iteration Algorithm [7]. This approach is depicted in Fig. 1.2 . 


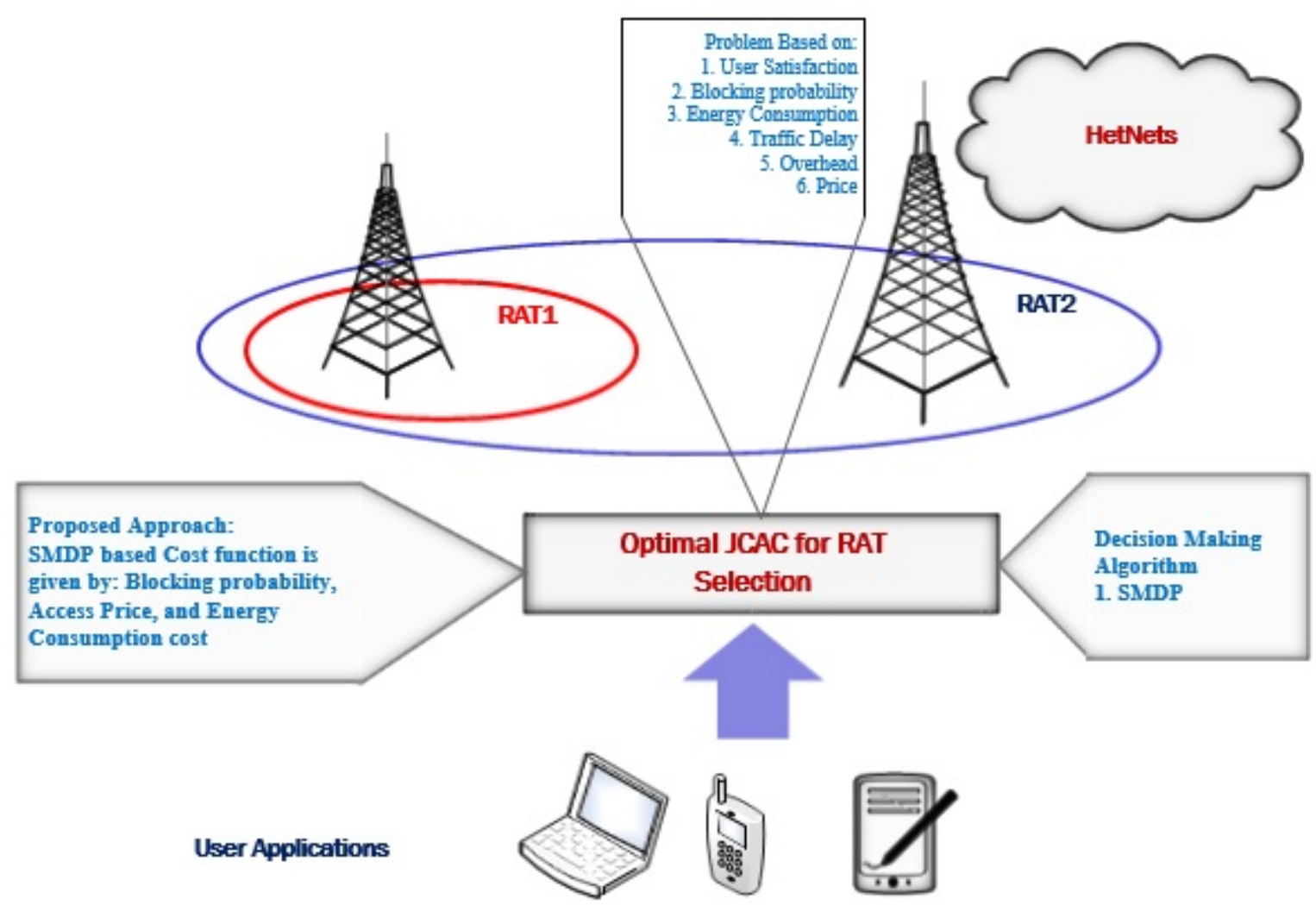

Figure 1.2: Our approach

\subsection{Thesis Contributions}

Our Contributions are two fold:

- We analyze the SMDP framework proposed in [5] and propose a novel JCAC based decision making strategy for initial RAT selection in HetNets composed of two colocated RATs.

- We validate our proposed RAT selection method by simulations using various network scenarios and settings, proving its effectiveness. 


\subsection{Thesis Organization}

The thesis is organized as follows:

- In Chapter 2, we overview HetNets and resource management in such networks.

- In Chapter 3, the SMDP model as well as the formulation of the optimization problem are presented.

- In Chapter 4, we describe the simulation results under various system configurations, scenarios, and performance metrics.

- In Chapter 5, we conclude our work. 


\section{Chapter 2}

\section{Background and Literature Review}

This Chapter sheds some light on the notion of HetNets and resource management in such networks. Few representative works on the problem of RAT selection in HetNets are discussed.

\subsection{Heterogeneous Wireless Networks}

Heterogeneous Wireless Networks (HetNets) is a mixture of co-existing radio access technologies (RATs) such as WiMax, WiFi, Bluetooth, Zigbee, IEEE802 WLANs that are expected to run in a balancing fashion. For example a HetNet composed of IEEE 802.11 WLANs and 3G/4G cellular systems may be used to provide higher bandwidth services over a wider geographic area. Indeed, HetNets uses different access technologies (i.e. radio interfaces) with overlapping coverage and different network architectures, protocols for transport, routing and mobility management. 
HetNet is a network with complex interoperation between macrocell, small cell, and in some cases WiFi network components, which are used together to provide a mixture of coverage, with handoff capability between network components [8]. The motivation of this approach was to combine several advantages such as low cost and high bandwidth. In HetNet, every network has its own characteristics based on the service demands originated from the users, (which include low-data rate, high-data rate, voice, multimedia, access technology, capacity, security, energy consumption, delay, coverage, and access cost) [9]. One of the most important features is that some of the wireless access networks are overlapped with each others in such a way that a multi-layer structure is naturally built, which matches various design purposes such as:

- Aggregating the system coverage: for example, by combining WiMAX and cellular mobile networks, a large geographical area can be covered.

- Scaling large volume data: networks can significantly help to scale large volume data distribution.

- Enhancing the system volume: the whole system may support many users by combining the capacity of each wireless access network within the proposed coverage region. This way, the blocking probabilities of new or handoff calls can be reduced, for example, by proposing alternative access points during overload situations [10], [11].

- Improving the user's satisfaction based on access costs: although some endusers may want to pay high prices for wireless access, most of them would 
prefer to get the lowest cost at a certain QoS level [12]. As a result, one of the most important design criteria in HetNets is choosing the price strategy.

To adopt these networks, and make use of their features, a system user needs to equip a multi-interface device capable of sensing and connecting the access network.

\subsection{Radio Resource Management in Heterogeneous Wire- less Networks}

The main objective of HetNets is to distribute global services to the users. However, the different networks that composed HetNets vary significantly in terms of network coverage, access cost, data transmission rate, and service class.

Due to the emergence of new wireless access technologies such as GSM, WiMax, UTMS/CDMA, LTE, Ham Radio, to name a few, and the explosion of mobile data services, HetNets is expected to become de facto the network of choice for many mobile network operators (MNOs) since a HetNet has the capability to use macro and various types of smaller cells (such as micro, pico and femto), along with the above-mentioned technologies together to offer wireless services while expanding their coverage. To acquire full benefit of the use of HetNet, one of the mandatory requirements is the design of an appropriate radio resource management (RRM) for such network. This task is difficult due to the fact that wireless networks that composed the HetNet may vary in terms of network coverage, access cost, data transmission rate, service class support [13], to name a few criteria. For this 
reason, traditional RRM schemes for wireless networks may no longer be applied to HetNets. Recent representative research works that address the problem of RRM in HetNets are [14], [15], [16], [17], [18]

In [14], Erturk et al. proposed a heterogeneous network composed of femtocells deployed within a macrocell network and a quality-of-service (QoS)- oriented fairness metric which captures important characteristics of tiered network architectures. Using homogeneous Poisson processes, the sum capacities in such networks are expressed in closed form for co-channel, dedicated channel, and hybrid resource allocation methods. They also proposed a resource splitting strategy that simultaneously considers capacity maximization, fairness constraints, and QoS constraints.

In [15], Liu et al. proposed almost blank resource block (ABRB) for interference control in HetNet. When an ABRB is scheduled in a macro BS, a resource block (RB) with blank payload is transmitted and this eliminates the interference from this macro BS to the pico BSs. They also studied a two timescale hierarchical radio resource management (RRM) scheme for HetNet with dynamic ABRB control. The long term controls, such as dynamic $\mathrm{ABRB}$, are adaptive to the large scale fading at a RRM server. The short term control (user scheduling) is adaptive to the local channel state information within each BS to exploit the multi-user diversity.

In [16], Rafie proposed a Multi-RAT small cells radio resource management. Multi-RAT RRM can bring significant benefits in the heterogeneous network including load balancing, interference distribution, reduction of unnecessary handovers and reduction of dropping/blocking probability in both licensed and unlicensed 
spectrum. Although the Multi-RAT RRM algorithms do not need to be standardized, the issue of the interoperability between multi-vendors and multi-RAT RANs could be addressed through a set of defined (open) interfaces.

In [17], Saraee et al. presented a cooperative JRRM method that can be used to integrate various different RATs so as to increase the coverage of each of them. In their scheme, the resource management responsibility is distributed among the network and the user based on a set of users preferences. A prototype implementation of their scheme is presented, validating its effectiveness in terms of handoff frequency and quality of users service.

In [18], Piamrat et al introduced two RRM schemes have been introduced which are suitable for HetNets, namely, the Common RRM (denoted CRRM) [2] and the Joint RRM (denoted JRRM) [4]. These RRM frameworks have been designed to achieve the following tasks: (1) Determining whether an incoming service request (from the user) should be accepted or blocked; and (2) Choosing in which of the cells an incoming service request should be accommodated. In particular, the JRRM scheme is made of three main modules, namely, (1) the Resource Monitoring module - which gathers the information from the users and the wireless networks composing the HetNet, then use these information to keep track of the active resources; (2) the Decision Making module - whose role is to select a suitable wireless network (within the pool of wireless networks composing the HetNet) that can handle the users request for connection and to allocate the necessary bandwidth for that connection; and (3) the Decision Enforcement module - which executes the decision made in Step 2. A more detailed description of each module 
can be found in [18]. This thesis makes use of the JRRM approach for designing a RAT selection mechanism in HetNets. Details on the design steps are described in Chapter 3.

\subsection{Literature Review}

This section overviews few representative RAT selection schemes for HetNets that are relevant to our thesis scope.

In [19], Falowo et al. suggested a dynamic RAT selection algorithm for allocating a multi-mode terminal with a single call to the best fit for RAT in the HetNets. They proposed an algorithm that is shown to save battery power consumption by selecting only one RAT at a time for multiple calls from a multi-mode terminal. However, their algorithm fails to treat each call when multiple calls originated from a multi-mode terminal are admitted into different RATs.

In [20], Haldaret et al. investigated a network and channel selection method in heterogeneous cognitive wireless network which is used by a cross-layer architectural framework. Their framework is classified by the users application, based on an Analytic Hierarchy Process algorithm.

In [21], Perez-Romero et al. introduced a RAT selection algorithm that uses criteria such as traffic load per RAT, service class, node speed, to name a few. To decide which RAT is suitable for handling the user's incoming request based on predefined policies.

In [22], Olabisi et al. described a joint call admission control protocol in which the RAT selection is achieved by implementing a mechanism that involves balanc- 
ing the traffic load in each RAT and adapting the bandwidth allocation of each RAT upon arrival of a new incoming request from the user.

In [23], a RAT selection algorithm is proposed in which a fuzzy-based approach is implemented, with the goal to achieve load balancing of the traffic among the RATs. The applicability of the proposed scheme relies on the availability of the targeted RAT to release enough bandwidth that can be used to accommodate the incoming user data.

In [24], Lucas et al. introduced a JRRM-based technique that utilizes the network load and the user fairness policy as decision criteria to select the best possible RATs that can accommodate the incoming request from the user.

In [25], Porjazoski et al. introduced a Markov decision-based RAT selection method that considers handover calls based on criteria such as user mobility, service type, and network load. Their proposed two-dimensional Markov chain method is shown to be superior to existing single or two criteria RAT selection techniques.

In [26], Mohamed et al. proposed a RAT selection algorithm where the decision maker is based on a multi-variable objective function that involves some weighted criteria such as user preferences, velocity, QoS. In their scheme, no formal method is proposed to determine the aforementioned weights.

In [27], $\mathrm{Si}$ et al. proposed a RAT selection method that uses the network access price and a multimedia distortion (QoS) as criteria to determine the best possible RAT to handle the user's incoming request. The optimal policy is achieved by means of a stochastic optimization problem that is designed according to the abovementioned criteria. 
In [28], Kosmidesa et al. proposed a network selection method that allocates terminals to the best suitable RAT in HetNets based on user's and provider's preferences. The decision is made according to three utility-based optimization functions that are solved using Branch and Bound, Greedy and strip packing techniques.

In [29], Modeas et al. proposed a policy-based RAT selection technique that takes into account the user moving speed, the network load and resource conditions, and the user preference as criteria to decide the most desirable RAT from the user perspective.

In [30], AL Sabbagh et al. overviews some Common Radio Resource Managementbased RAT selection mechanisms for HetNets that support the provision of QoS and radio resource utilization. Based on their investigation, a RAT selection method is designed based on multicriteria such as type of service handled by the HetNet, measurements from local RRM of the avilable RATs, operator policy, requested QoS from the users, interference conditions, load at each RAT, cost of requested services, to name a few.

Unlike the above representative works, this thesis considers a cost function that optimizes three criteria namely, the blocking cost, the access cost, and the energy consumption cost. Most of the works in literature use the MDP based framework to formulate the problem studied in this thesis, where the decision is based on fixed epochs. However, in this thesis, an SMDP-based model is introduced to address the same problem [5], but this time, under various different decision making strategies. In our proposed SMDP system dynamic, arrivals and departures are modeled by 
exponential distributions, in such a way that the times between decision epochs are not fixed (but rather are random). 


\section{Chapter 3}

\section{Methodology}

\subsection{System Model}

We consider a HetNet composed of two co-located RATs serving two types of service classes. The $j^{\text {th }} \operatorname{RAT}(j=1,2)$ has $N_{j}$ radio resources. In addition, the service class- 1 call is the higher priority class call with pre-emptive right. It arrives into the system according to a poisson process with parameter $\lambda_{1}$. Service class-2 is the lower priority call. It arrives into the system according to a poisson process with parameter $\lambda_{2}$. Each incoming service call is served by allocating the required amount of resources from one of the available RATs. Based on how the radio interface is implemented, RAT technologies such as CDMA, OFDM, FDMA, TDMA, to name a few, can be used, and the system capacity can be implemented by its effective or equivalent bandwidth [5]. The optimal RAT selection method will decide whether the call will be accepted or rejected based on when an incoming service connection needs to access the HetNets. It also decides which RAT will 
accept the call. In HetNets, each class is categorized by its bandwidth requirement, arrival distribution, and channels holding time. The $i^{\text {th }}$ service connection $(i=1,2)$ arrives in the system according to a poisson process with parameter $\lambda_{i}$ and it require $b_{i}$ radio resources. The traffic intensity is defined by $\rho_{i}=\lambda_{i} / \mu_{i}$, where the channel holding time is based on the connection duration and the residence time which follow an exponential distribution with mean rate $\mu_{i}$.

\subsection{Problem Formulation}

To formulate the RAT selection problem in HetNet, we introduce a SMDP framework.

\subsubsection{SMDP Model}

The SMDP model is based on the following elements: the state space, the decision epochs and the actions, the expected time until the next decision epoch, the transition probabilities, and the cost function.

\subsubsection{State Spaces}

The state spaces of the SMDP model are defined by the five-tuple.

$$
S=\left(n_{11}, n_{21}, n_{12}, n_{22}, e\right)
$$

where the following constraints are associated with each RAT:

$$
0 \leq n_{i j} \leq\left\lceil N_{j} / b_{i}\right\rceil, e=\left[\begin{array}{lll}
0 & 1 & 2
\end{array}\right]^{T}
$$


where $n_{i j}$ is the number of calls of type $i$ connection in RAT $-\mathrm{j}, N_{j}$ is the capacity of RAT-j, and $b_{i}$ is the bandwidth required by type $i$ call connection. $e=0$ means the departure of a connection, $e=1$ means the arrival of call connection of type 1 and $e=2$ means the arrival of call connection of type 2 .

\subsubsection{Decision Epochs With Actions}

The JRRM policy relies on the following possible actions:

- Blocking of a call (represented as B)

- Accepting a call in RAT1 (represented as AR1)

- Accepting a call in RAT2 (represented as AR2)

For each state $x \in S$, the controller can select one of the following possible decisions and actions based on the arrival of a new call:

$$
A(x)=\left\{\begin{array}{cl}
A R 1, & \text { if } e=1,2 \text { and } b_{(i=(1,2))}+b_{1} n_{11}+b_{2} n_{21} \leq N_{1} \\
A R 2, & \text { if } e=1,2 \text { and } b_{(i=(1,2))}+b_{1} n_{12}+b_{2} n_{22} \leq N_{2} \\
B, & \text { if } e=0,1,2
\end{array}\right.
$$

\subsubsection{Expected Time Until The Next Decision Epoch}

When the system is in state $x \in S$ and the action $a \in A(x)$ is taken, the expected time until the next decision epoch $\tau(x, a)$ is obtained as:

$$
\tau(x, a)=\left(\lambda_{1}+\lambda_{2}+n_{11} \mu_{1}+n_{21} \mu_{2}+n_{12} \mu_{1}+n_{22} \mu_{2}\right)^{-1}
$$


where, $\lambda_{1}$ is the arrival rate for type- 1 call, $\lambda_{2}$ is the arrival rate for type- 2 call, $n_{i j}$ is the number of calls of type $i$ connection in RAT-j. $\mu_{1}$ and $\mu_{2}$ is the channel holding time for service class- 1 and service class- 2 respectively.

\subsubsection{Transition Probabilities}

The transition probabilities between the system states are intended to identify the state dynamic. Assuming that the transition probability at the next decision epoch is $t p(x, y, a)$, the system will be in state $y \in S$ if action $a \in A(x)$ is chosen in state $x$. Also, the expected time until the next decision epoch is $\tau(x, a)$ if the action $a \in A(x)$ is chosen in state $x$. The transition probabilities are obtained as follows:

Case-1: Arrival of type-1 calls

$$
t p(x, y, a)=\left\{\begin{aligned}
\lambda_{1} \tau(x, a), x=\left(n_{11}, n_{21}, n_{12}, n_{22}, 1\right) \Rightarrow y=\left(n_{11}+1, n_{21}, n_{12}, n_{22}, e\right), & \text { if } \quad a=A R 1 \in A(x) \\
\lambda_{1} \tau(x, a), x=\left(n_{11}, n_{21}, n_{12}, n_{22}, 1\right) \Rightarrow y=\left(n_{11}, n_{21}, n_{12}+1, n_{22}, e\right), & \text { if } a=A R 2 \in A(x) \\
\lambda_{1} \tau(x, a), x=\left(n_{11}, n_{21}, n_{12}, n_{22}, 1\right) \Rightarrow y=x, & \text { if } a=B \in A(x)
\end{aligned}\right.
$$

Case-2: Arrival of type-2 calls

$$
\operatorname{tp}(x, y, a)=\left\{\begin{aligned}
\lambda_{2} \tau(x, a), x=\left(n_{11}, n_{21}, n_{12}, n_{22}, 2\right) \Rightarrow y=\left(n_{11}, n_{21}+1, n_{12}, n_{22}, e\right), & \text { if } \quad a=A R 1 \in A(x) \\
\lambda_{2} \tau(x, a), x=\left(n_{11}, n_{21}, n_{12}, n_{22}, 2\right) \Rightarrow y=\left(n_{11}, n_{21}, n_{12}, n_{22}+1, e\right), & \text { if } a=A R 2 \in A(x) \\
\lambda_{2} \tau(x, a), x=\left(n_{11}, n_{21}, n_{12}, n_{22}, 2\right) \Rightarrow y=x, & \text { if } a=B \in A(x)
\end{aligned}\right.
$$


Case-3: Departures of calls

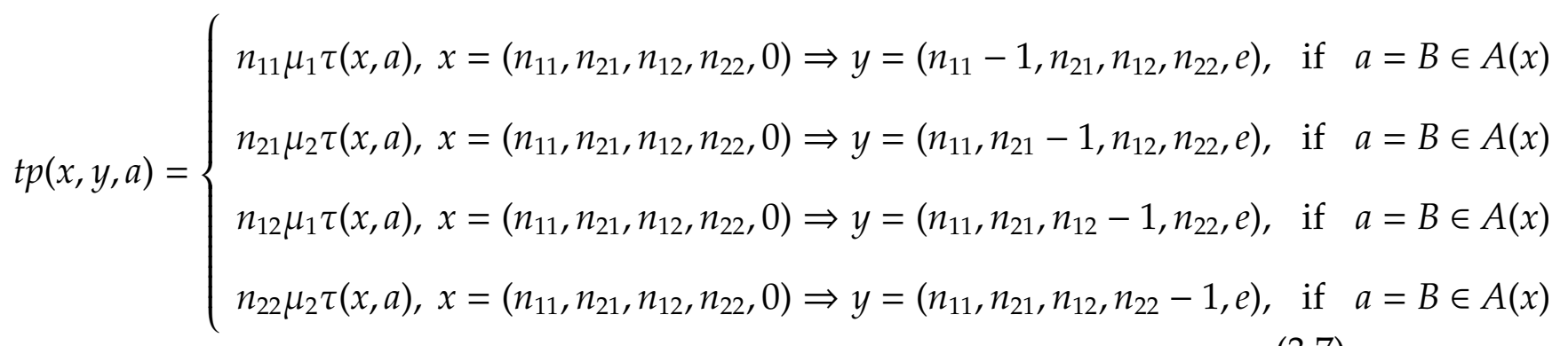

\subsubsection{Cost Function}

In our JCAC -based scheme, the decision of accepting or rejecting a new service request is dependent on a cost function $\mathrm{C}(\mathrm{x}, \mathrm{a})$, where state $x \in S$ and the action $a \in A(x)$, defined as:

$$
C(x, a)=\omega_{1} g_{b c}(x, a)+\omega_{2} g_{a c}(x, a)+\omega_{3} g_{e c}(x, a)
$$

where $\omega_{1}$ is the weight associated to the blocking cost function $g_{b c}(x, a), \omega_{2}$ is the weight associated to the access cost function $g_{a c}(x, a)$, and $\omega_{3}$ is the weight associated to the energy consumption cost $g_{e c}(x, a)$, such that with $\omega_{1}+\omega_{2}+\omega_{3}=1$.

The values assigned to these weight functions can be adjusted according to the MNO's need. In equation 3.8, the cost function $g_{b c}(x, a)$, is incurred whenever an incoming traffic is blocked by the system. It is defined as:

$$
g_{b c}(x, a)=\left\{\begin{array}{rc}
B C_{i}, e=1,2 & \text { and } a=B \\
0, & \text { otherwise }
\end{array}\right.
$$

where $B C_{i}$ is the blocking cost of the $i^{\text {th }}$ service class. The network access cost 
function $g_{a c}(x, a)$ is determined as:

$$
g_{a c}(x, a)=\left\{\begin{array}{r}
\frac{A_{i j}-\min _{1 \leq i, j \leq 2}\left(A_{i j}\right)}{\max _{1 \leq i, j \leq 2}\left(A_{i j}\right)-\min _{1 \leq i, j \leq 2}\left(A_{i j}\right)}, \text { if } e=1,2 \text { and } a=A R_{j} \\
0, \text { otherwise }
\end{array}\right.
$$

where $A_{i j}$ is the access cost for the service of type $i$ for RAT- $j$. The energy consumption cost $g_{e c}(x, a)$ represents the energy required to operate a base station or the access point. It is defined as:

$$
g_{e c}(x, a)=\left\{\begin{array}{r}
\frac{E_{j}-\min _{1 \leq j \leq 2}\left(E_{j}\right)}{\max _{1 \leq j \leq 2}\left(E_{j}\right)-\min _{1 \leq j \leq 2}\left(E_{j}\right)}, \text { if } e=1,2 \quad \text { and } a=A R_{j} \\
0, \text { otherwise }
\end{array}\right.
$$

where, $E_{j}$ is the energy consumed by the $j^{\text {th }}$ RAT.

\subsubsection{JCAC Policy}

A JCAC policy is a vector of an $n$-tuple identifying for each state the action to be selected in that state. It is assumed that the policy does not change in time and in a give state. It should be noticed that for a MDP model with finite state space and finite action sets, an optimal policy exists which is stationary and deterministic. Such policy is an application from $\mathrm{S}$ to $\mathrm{A}$, where each state $x \in S$ is associated with an action $x \in A$ such that:

$$
\forall x \in S, R_{x} \in A(x)
$$

It should be noted that the SMDP model with transition probabilities $t p\left(x, y, R_{x}\right)$ is a traditional continuous time Markov chain. In order to derive the optimal JCAC policy, it is required to transform the continuous time SMDP model into a discrete time MDP model. To do this, we utilize the value iteration algorithm proposed in [7], which is depicted in Algorithm.1] 


\section{Algorithm 1 Value-Iteration Algorithm [7]}

\section{Step 0: Initialization}

Select $V_{0}(i)$ such that $0 \leq V_{0}(i) \leq \min _{a}\{c(i, a) / \tau(i, a)\}$ for all $i$.

Select a number $\tau$ where $0<\tau<\min _{i, a} \tau(i, a)$.

Let $n=1$

Step 1: Value-iteration step

Compute the function $V_{n}(i), i \in I$ using

$$
V_{n}(i)=\min _{a \in A(i)}\left[\frac{c(i, a)}{\tau(i, a)}+\frac{\tau}{\tau(i, a)} \sum_{j \in I} p(i, j, a) V_{n-1}(j)+\left(1-\frac{\tau}{\tau(i, a)}\right) V_{n-1}(i)\right]
$$

Let $R(n)$ be a fixed policy whose actions minimize the right-hand side of Equation 3.13 .

Step 2: Compute the bounds $m_{n}$ on the minimal cost using

$$
m_{n}=\min _{j \in I}\left\{V_{n}(i)-V_{n-1}(i)\right\}, \quad M_{n}=\max _{j \in I}\left\{V_{n}(i)-V_{n-1}(i)\right\}
$$

Step 3: Stopping condition. The algorithm is stopped when policy $R(n)$ is obtained such that $0 \leq\left(M_{n}-m_{n}\right) \leq \epsilon m_{n}$ where $\epsilon$ is a prescribed accuracy number. Otherwise, go to step 4.

Step 4: Continue

$n=n+1$ and go to step 1 .

More precisely, if $x, y \in S$ and $a \in A(x)$, select a number $\tau$ such that:

$$
0<\tau \leq \min _{x, a} \tau(x, a)
$$

Based on this selection, the system executes the following transformations: 


$$
\begin{gathered}
\bar{S}=S \\
\bar{A}(x)=A(x), x \in \bar{S} \\
\bar{C}(x, a)=\frac{C(x, a)}{\tau(x, a)}, x \in \bar{S} \text { and } a \in \bar{A}(x) \\
\bar{p}(x, y, a)=\left\{\begin{array}{l}
\frac{\tau}{\tau(x, a)} p(x, y, a), x \neq y, x \in \bar{S} \text { and } a \in \bar{A}(x) \\
\frac{\tau}{\tau(x, a)} p(x, y, a)+\left[1-\frac{\tau}{\tau(x, a)}\right], x=y, x \in \bar{S} \text { and } a \in \bar{A}(x)
\end{array}\right.
\end{gathered}
$$

where $\bar{i}$ denotes the transformed module. The optimal JCAC policy $R(n)$ is then obtained by transforming the continuous time SMDP model into a discrete time MDP model, whose average cost function is determined as:

$$
0 \leq \frac{g_{i}(R(n))-g^{*}}{g^{*}} \leq \epsilon
$$

where $g^{*}$ represents the minimal average cost for each time unit. 


\section{Chapter 4}

\section{Performance Evaluation}

\subsection{Simulation Tool and Parameters}

For simulation purpose, the discrete time Markov model can only be resolved for small values of $N_{j},(j=1,2)$ which is dependent on the state-space explosion problem as shown in the equation 3.1. Therefore, we used a numerical value for our simulation model, where $N_{1}$ is equal to 24 channels and $N_{2}$ is equal to 12 channels.

We simulate the model by using Borland $\mathrm{C}++\mathrm{v} 5$ for Windows 7

We also use $1.0 e-12$ precision value for each system configuration. In this section, RAT1 and RAT2 are considered as representative of GSM and UMTS technologies. In addition, two service classes (class-1 and class-2 are considered). Parameters that are fixed in our simulations are captured in Table 4.1. 
Table 4.1: Fixed parameters values for simulation

\begin{tabular}{cc|cc|cc}
\hline \hline Parameter & Value & Parameter & Value & Parameter & Value \\
\hline$N_{1}$ & 24 channels & $\mu_{1}$ & $1 / 120$ s (voice) & $E_{1}$ & $3802 \mathrm{~W}$ \\
$N_{2}$ & 12 channels & $\mu_{2}$ & $1 / 120$ s (voice) & $E_{2}$ & $300 \mathrm{~W}$ \\
$A_{11}$ & 20 & $A_{12}$ & 10 & $B C_{1}$ & 1.0 \\
$A_{21}$ & 10 & $A_{22}$ & 5 & $B C_{2}$ & 0.8 \\
\hline
\end{tabular}

\subsection{Performance Measurement}

The following performance metrics are considered for evaluating the system's performance. The mean carried traffic $M_{e}^{a}$, computed as:

$$
M_{e}^{a}=\sum_{x \in S ; e=1,2 ; a=A R 1, A R 2 \in A(x)}\left(\sum_{j=1}^{2} \lambda_{j}+\sum_{j=1}^{2} \sum_{i=1}^{2} n_{i j} \mu_{i}\right) \pi_{x}
$$

where $\pi_{x} ; x \in S$ is the continuous time Markov chain steady state probability distribution in the optimal policy, $n_{i j}$ is the number of calls of type $i$ connection for RAT $-j$.

- The connection blocking probability $P b_{i}$ of a new $i^{\text {th }}$ service class is defined as:

$$
P b_{i}=1-\frac{M_{i}^{a}}{\lambda_{i}}
$$

It should be noted that for the MNO benefits, $P b_{i}$ must be as low as possible.

- The bandwidth utilization $U_{j}$ of the $j^{\text {th }} \mathrm{RAT}$ is determined as:

$$
U_{j}=\frac{1}{N_{j}} \sum_{x \in S ; a \in A(x) ; \forall i ; \forall j ; n_{i j}>0} b_{i} n_{i j} \pi_{x}
$$

where $b_{i}$ is the bandwidth of type $i$ call connection, $N_{j}$ is the capacity of RAT- $j$, and $n_{i j}$ is the number of calls of type $i$ connection for RAT- $j$. 


\subsection{Simulation Results}

We considered the scenarios showed in Table 4.2, 4.3, 4.4, 4.5 ,

Table 4.2: Scenarios-I

\begin{tabular}{cc|cc}
\hline Parameter & Value & Parameter & Value \\
\hline Bandwidth for service class-1 $\left(b_{1}\right)$ & 2 channels & Weight-1 of Blocking Cost $\left(\omega_{1}\right)$ & 0.6 \\
Bandwidth for service class-2 $\left(b_{2}\right)$ & 1 channel & Weight-2 of Access Price Cost $\left(\omega_{2}\right)$ & 0.2 \\
& & Weight-3 of Energy Consumption $\operatorname{cost}\left(\omega_{3}\right)$ & 0.2 \\
\hline
\end{tabular}

Table 4.3: Scenarios-II

\begin{tabular}{cc|cc}
\hline Parameter & Value & Parameter & Value \\
\hline Intensities for service class-1 $\left(\rho_{1}\right)$ & 7 channels & Weight-1 of Blocking Cost $\left(\omega_{1}\right)$ & 0.6 \\
Intensities for service class-2 $\left(\rho_{2}\right)$ & \multirow{2}{*}{3 channel } & Weight-2 of Access Price Cost $\left(\omega_{2}\right)$ & 0.2 \\
& & Weight-3 of Energy Consumption cost $\left(\omega_{3}\right)$ & 0.2 \\
\hline
\end{tabular}

Table 4.4: Scenarios-III

\begin{tabular}{cc|cc}
\hline Parameter & Value & Parameter & Value \\
\hline Bandwidth for service class-1 $\left(b_{1}\right)$ & 2 channels & Intensities for service class-1 $\left(\rho_{1}\right)$ & 7 \\
Bandwidth for service class-2 $\left(b_{2}\right)$ & 1 channel & Intensities for service class-1 $\left(\rho_{2}\right)$ & 3 \\
& & Weight-2 of Access Price $\left(\omega_{2}\right)$ & 0.2 \\
\hline
\end{tabular}

Table 4.5: Scenarios-IV

\begin{tabular}{cc|cc}
\hline Parameter & Value & Parameter & Value \\
\hline Bandwidth for service class-1 $\left(b_{1}\right)$ & 2 channels & Intensities for service class-1 $\left(\rho_{1}\right)$ & 5 \\
Bandwidth for service class-2 $\left(b_{2}\right)$ & 1 channel & Intensities for service class-1 $\left(\rho_{2}\right)$ & 3 \\
& & Weight-1 of Blocking $\left(\omega_{2}\right)$ & 0.6 \\
& & Weight-2 of Access Price $\left(\omega_{2}\right)$ & 0.2 \\
& & Weight-3 of Energy cost $\left(\omega_{2}\right)$ & 0.2 \\
\hline
\end{tabular}


- Scenario - I: The traffic intensity is defined as the number of calls received by the network elements in a unit area at a given time interval. In this scenario, the traffic intensity is varied and the impact of this variation on the blocking probabilities, the RAT utilization, and the optimal cost is studied respectively. The results are captured in Fig. 4.1(a) and (b) (Case of blocking probabilities), Fig. 4.2 (a) and (b) (Case of RAT utilization), and Fig. 4.3 (Case of optimal cost).

- Scenario - II: In this scenario, the bandwidth of each class of traffic is varied and the impact of this variation on the blocking probabilities, the RAT utilization, and the optimal cost is studied respectively. The results are captured in Fig. $4.4(\mathrm{a})$ and (b) (Case of blocking probabilities), Fig. 4.5/(a) and (b) (Case of RAT utilization), and Fig.4.6(Case of optimal cost).

- Scenario - III: In this scenario, the weight of energy consumption $\left(\omega_{3}\right)$ in the total cost function is varied and we study the impact of this variation on the blocking probabilities. This scenario is to demonstrate the relative importance of the energy consumption cost on the system performance. The results are captured in Fig. 4.7(a) and Fig. 4.7(b). We also studied the impact of this variation on the RAT utilization and the optimal cost respectively. The results are captured in Fig. $4.8(\mathrm{a})$ and (b), and Fig. 4.9 respectively.

- Scenario - IV: In this scenario, the channel holding time is varied and the impact of this variation on the blocking probabilities, the RAT utilization, and the optimal cost are studied respectively. The results are captured in 
Fig. 4.10 (Case of blocking probabilities), Fig. 4.11 (a) and (b) (Case of RAT utilization), and Fig. 4.12 (Case of optimal cost) respectively. Finally, we analyze the structure of the optimal JCAC policy. Our results are captured in Tables 4.6 to 4.9

\subsection{Simulation Results}

\subsubsection{Results for Scenario - I}

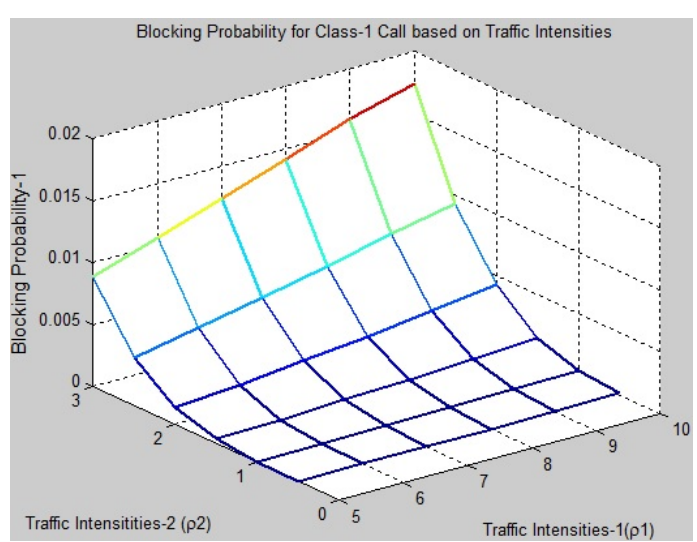

(a)

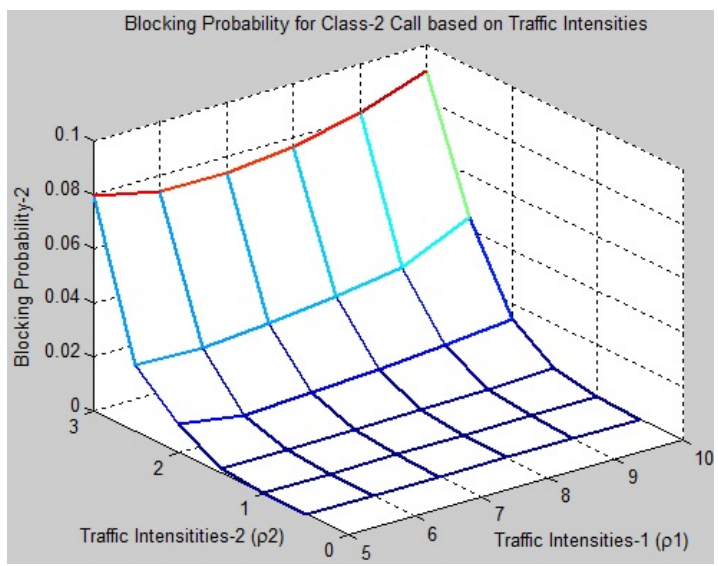

(b)

Figure 4.1: Scenario I - Blocking probability vs. Traffic intensities (a) Case of class-1 calls and (b) Case of class- 2 calls.

In Fig. 4.1 (a) and (b), it can be observed that when the traffic intensities increase, so are the blocking probabilities as well, for a fixed system capacity. It can be observed that more traffic of class- 1 are blocked compared to traffic of class2. The reason for this behavior is that class- 1 calls require more bandwidth than class-2 calls. 
In Fig. 4.2 (a) and (b), it can be observed that when the traffic intensities are smaller, the JCAC policy accepts more calls in the less energy - consumed RAT (i.e. RAT2 in this case), hence saving some energy. Due to this fact, the utilization of RAT2 is much better than that of RAT1. On the other hand, when the traffic intensities are higher, more calls are accepted in RAT1 (compared to RAT2), leading to a higher utilization of RAT1.

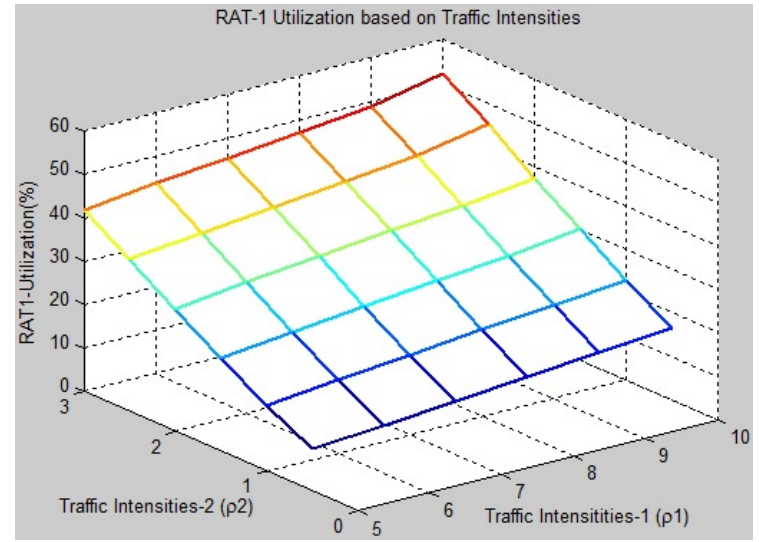

(a)

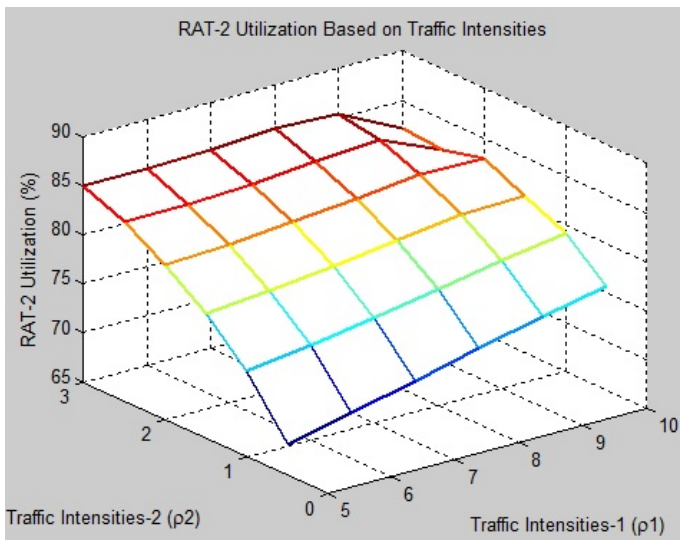

(b)

Figure 4.2: Scenario I - RAT utilization vs. Traffic intensities (a) Case of RAT1 and (b) Case of RAT2.

In Fig. 4.3, it can be observed that when the traffic intensities increase, the optimal cost also increases. This is attributed to the fact that the blocking probabilities of both calls do increase because there are less number of channels that are free to take new incoming calls. 


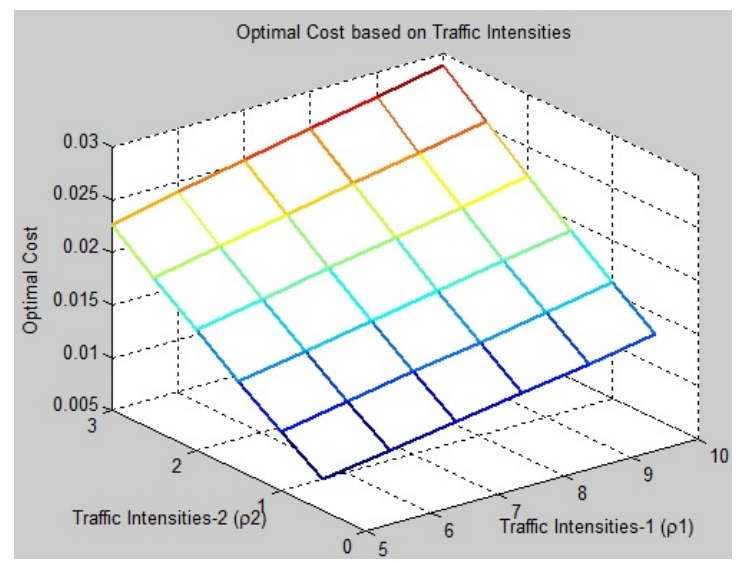

Figure 4.3: Scenario I - Optimal cost vs. Traffic intensities.

\subsubsection{Results for Scenario - II}

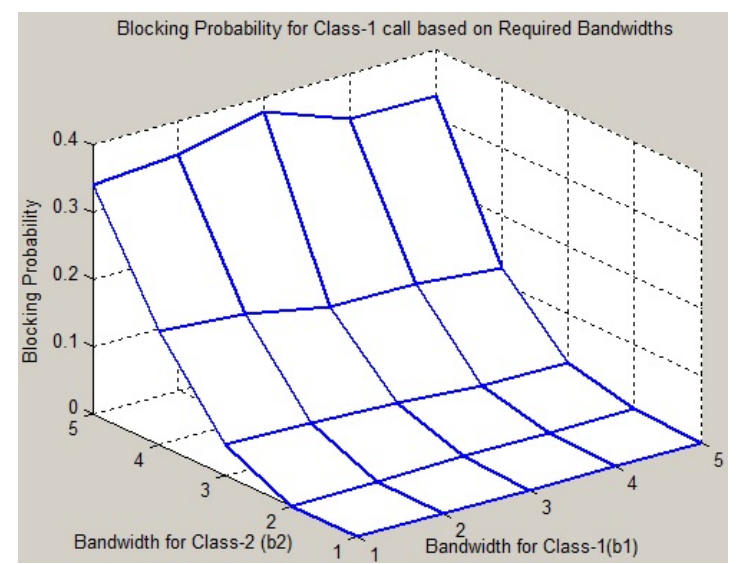

(a)

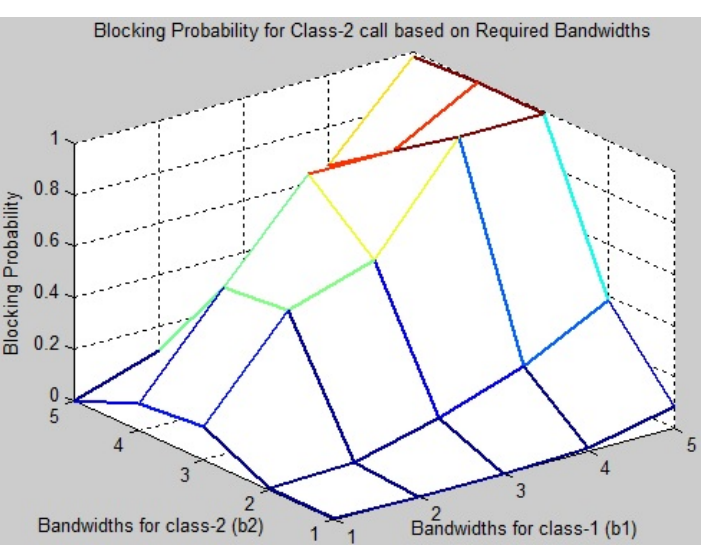

(b)

Figure 4.4: Scenario II - Blocking probability vs. Bandwidth (a) Case of class-1 call and (b) Case of class-2 call.

In Fig. 4.4(a) and (b), it can be observed that when the bandwidth of class-1 calls (resp. class-2 calls) increases, less vacant channels are available to accommodate new incoming calls, therefore the blocking probabilities of each traffic class become 


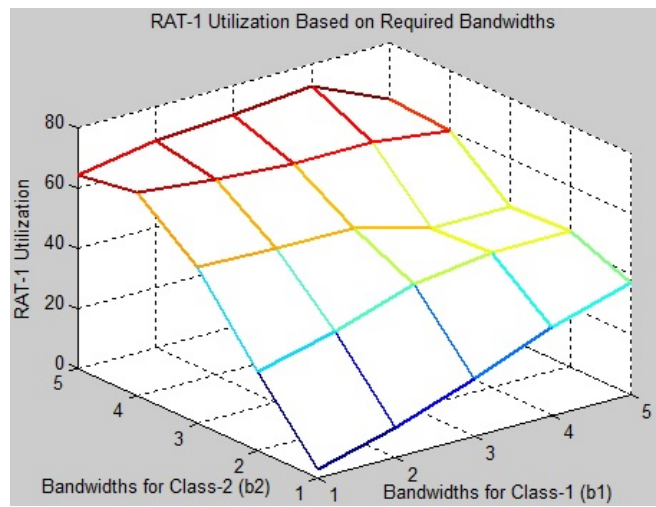

(a)

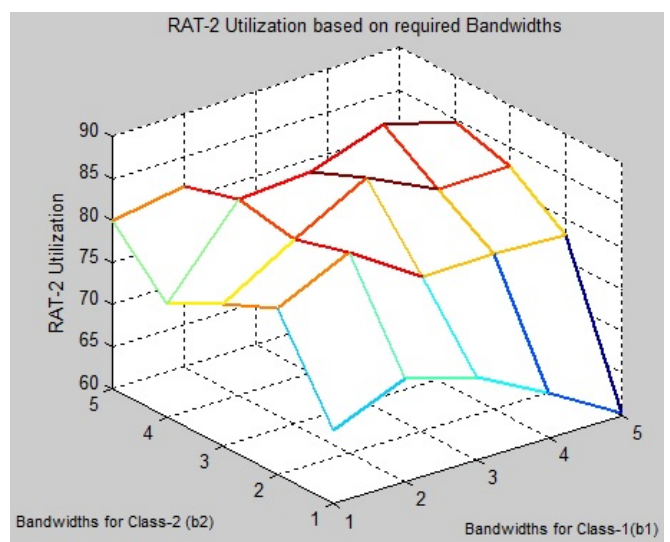

(b)

Figure 4.5: Scenario II - RAT utilization vs. Bandwidth (a) Case of RAT1 and (b) Case of RAT2

high, which was expected. Compared to the results on the blocking probabilities that were obtained in figure 4.1. This is attributed to the fact that class-2 traffic has lower priority compared to class-1 traffic.

In Fig. 4.5 (a) and (b) it can be observed that RAT2 utilization is much better than RAT1 utilization, especially when the bandwidth of each call is low. This is attributed to the fact that RAT2 consumes less energy compared to RAT1, thus it is more often chosen by the JCAC policy. However when the required bandwidth for each call increases, the optimal policy accepts more and more calls in RAT1 when RAT2 can no longer accommodate new incoming calls (i.e when it is fully utilized). 


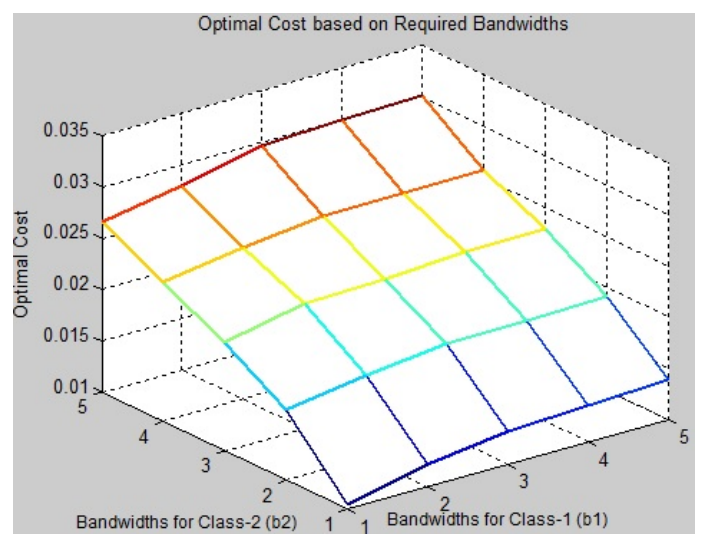

Figure 4.6: Scenario II - Optimal cost vs. Bandwidth.

In Fig. 4.6, it can be observed that when the required bandwidth for each class increases, the optimal cost also increases. This is attributed to the fact that the blocking probability increases when the required bandwidth increases. Thus, less channels remains on the systems to accommodate new incoming calls.

\subsubsection{Results for Scenario III}

In Fig. $4.7(\mathrm{a})$, it can be observed that when the weight of energy cost $\left(\omega_{3}\right)$ is set to a value in the range $[0.1,0.25]$, the optimal policy accepts incoming calls from both class- 1 and class-2. However, when $\omega_{3} \geq 0.25$, the optimal policy starts to reject both types of calls. The same behavior can be observed in Fig.4.7|(b), It can also be observed that more class- 1 calls are blocked by the optimal policy compared to class-2 calls. 


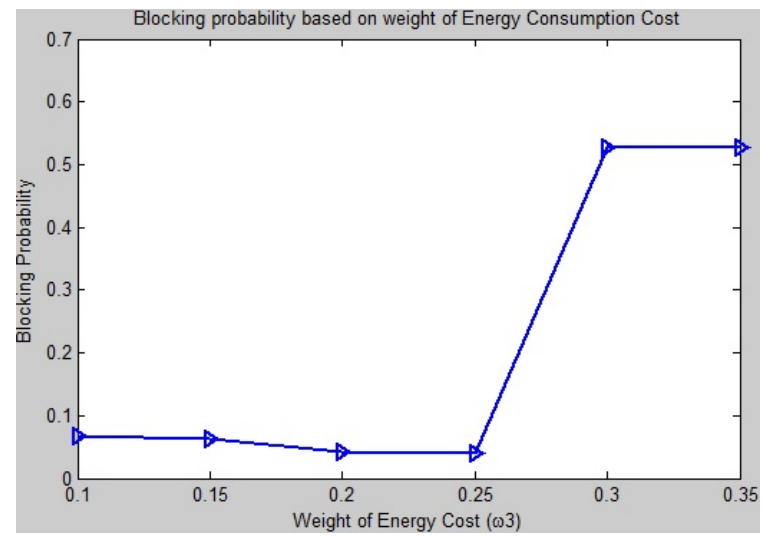

(a)

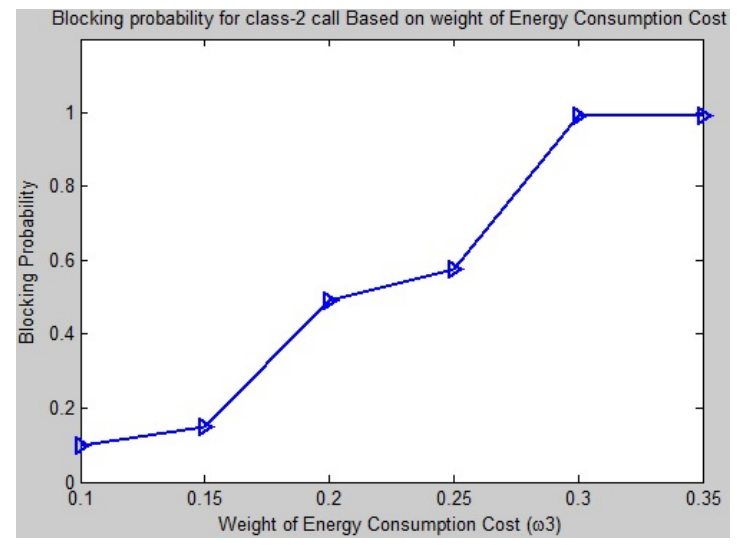

(b)

Figure 4.7: Scenario III - Blocking probability vs. Weight of energy consumption cost in total cost (a) Case of class- 1 calls and (b) Case of class- 2 calls. 


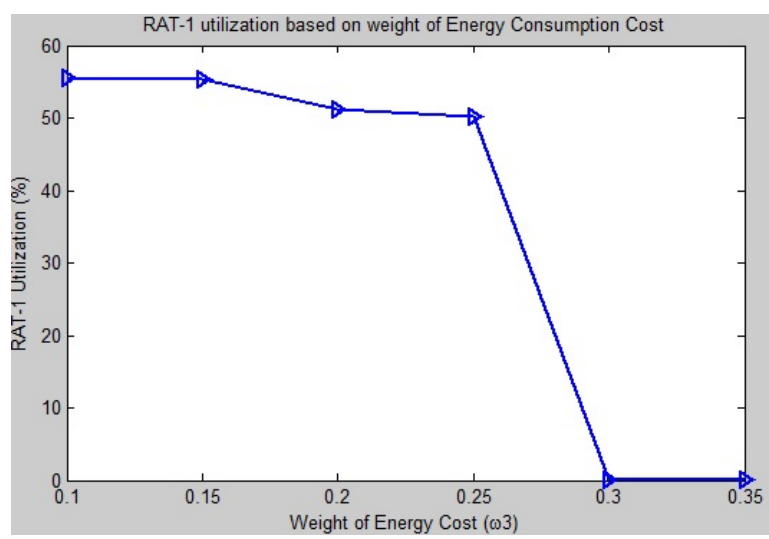

(a)

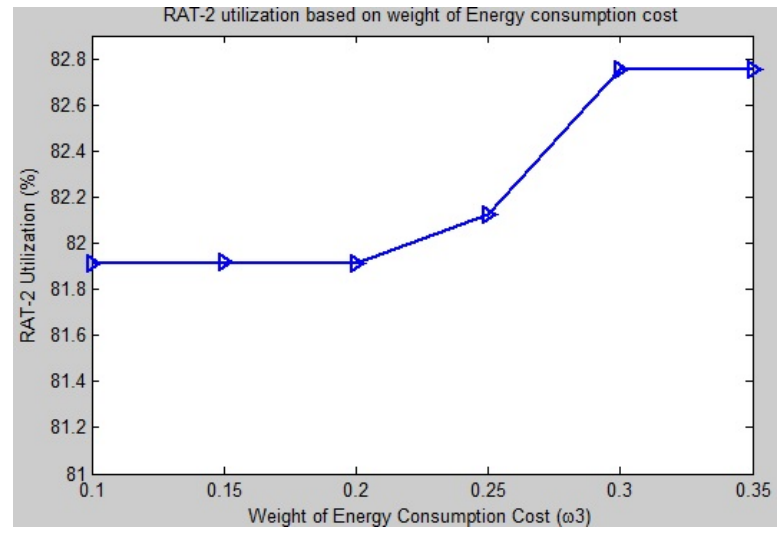

(b)

Figure 4.8: Scenario III - RAT utilization vs. Weight of energy consumption cost in total cost (a) Case of RAT1 and (b) Case of RAT2.

In Fig. 4.8 (a) and (b) , it can be observed that when the weight of access price $\left(\omega_{2}\right)$ is set to 0.2 (i.e $20 \%$ of the total network cost) and the weight of blocking cost $\left(\omega_{1}\right)$ and weight of the energy consumption cost $\left(\omega_{3}\right)$ are varied from 0.45 to 0.7 (i.e $(45 \%)$ to $(70 \%)$ and from $(10 \%)$ to $(35 \%)$, respectively, the JCAC policy utilizes more channels of RAT2 (81.9\%) compared to that of RAT1 (only 55\%). This is attributed to the fact that RAT1 necessities more energy consumption for its operations than RAT2 does. However, when the energy consumption cost $\left(\omega_{3}\right)$ is set to $(30 \%)$, 
RAT1 utilization reaches almost (0\%) Fig. 4.8 (a) whereas RAT2 channel utilization increases slightly Fig. $4.8(\mathrm{~b})$. This is due to the fact that more calls that should have been served by RAT1 are now handled by RAT2 Fig. 4.9, it can be observed that the optimal cost increases when the weight of the energy consumption cost $\omega_{3}$ is set to low values (from 0.1 to 0.3 ). When $\omega_{3}$ is set to a value greater than (i.e $\omega_{3} \geq 0.3$ ) (high values), the system starts blocking more calls in order to save energy.

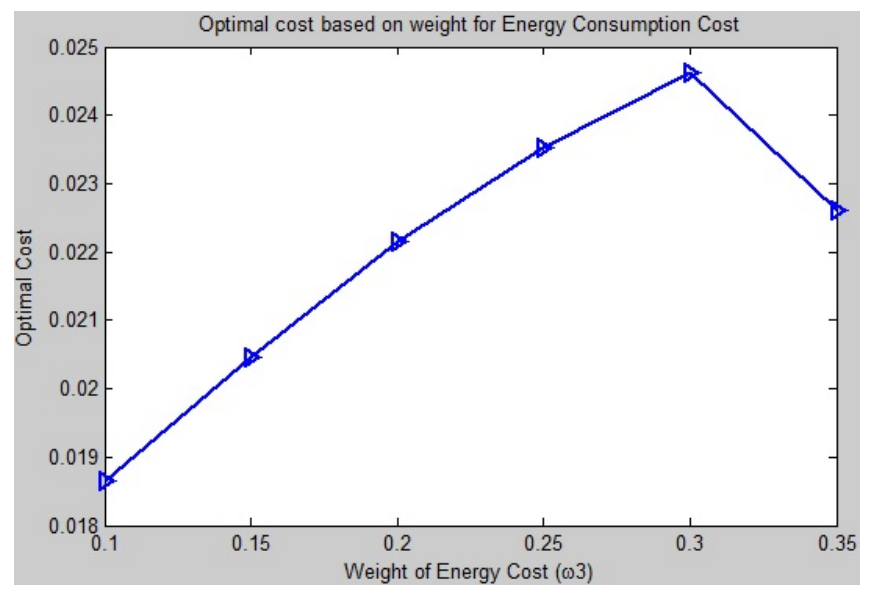

Figure 4.9: Scenario III - Optimal cost vs. Weight for energy consumption cost in total cost.

\subsubsection{Results for Scenario IV}

In Fig. 4.10, it can observed that when the channel holding time increases, the blocking probabilities also increases. This phenomenon is less pronounced for class- 1 calls compared to class- 2 calls. This is attributed to the fact that the system blocks class-2 traffic in less time because of its lower call intensity. 


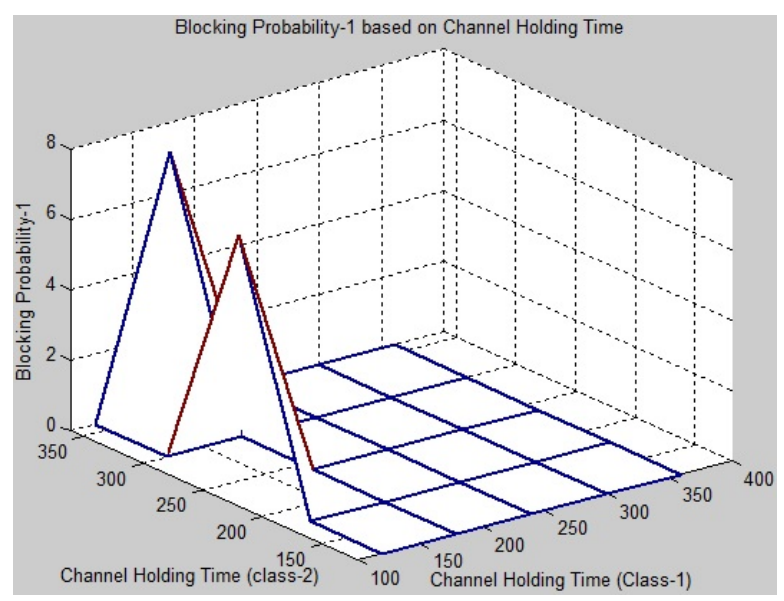

(a)

Figure 4.10: Scenario IV - Blocking probability vs. Channel holding time

In Fig. 4.11(a), it can be observed that the utilization of RAT1 is almost flat when it reaches $15 \%$ by servicing channel holding time 300 to 360 seconds for class- 1 . At the same time, RAT1 Utilization for class- 2 went down almost $0 \%$ when the channel holding time is 240 seconds and 360 seconds. In Fig. 4.11(b), it can be observed that the utilization of RAT2 is flat, when it reaches almost $60 \%$. At the same time, it fluctuates for different channel holding times in class-1 and class-2 (i.e. when the channel holding time is 240 seconds and 360 seconds, the RAT utilization becomes almost $0 \%$ in both class- 1 and class-2). 


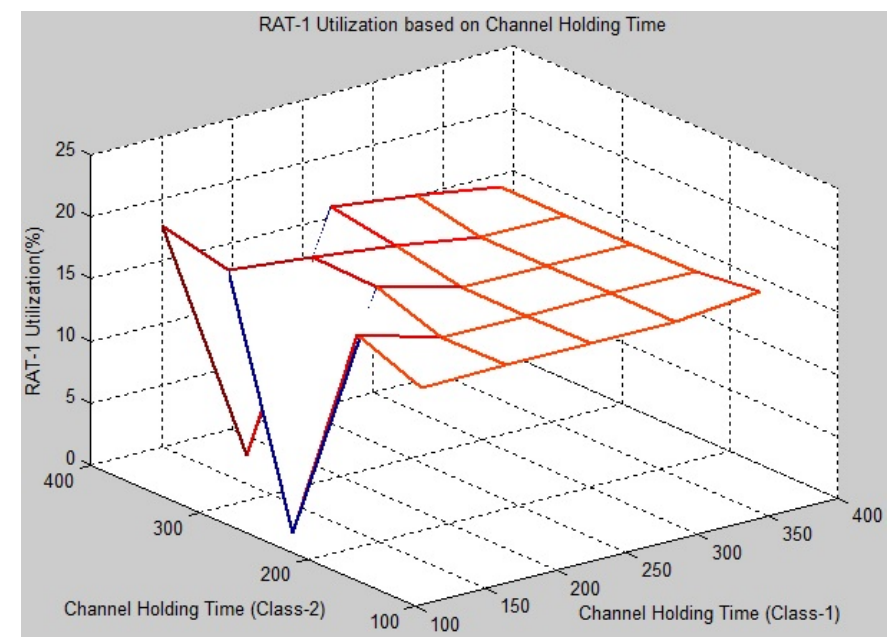

(a)

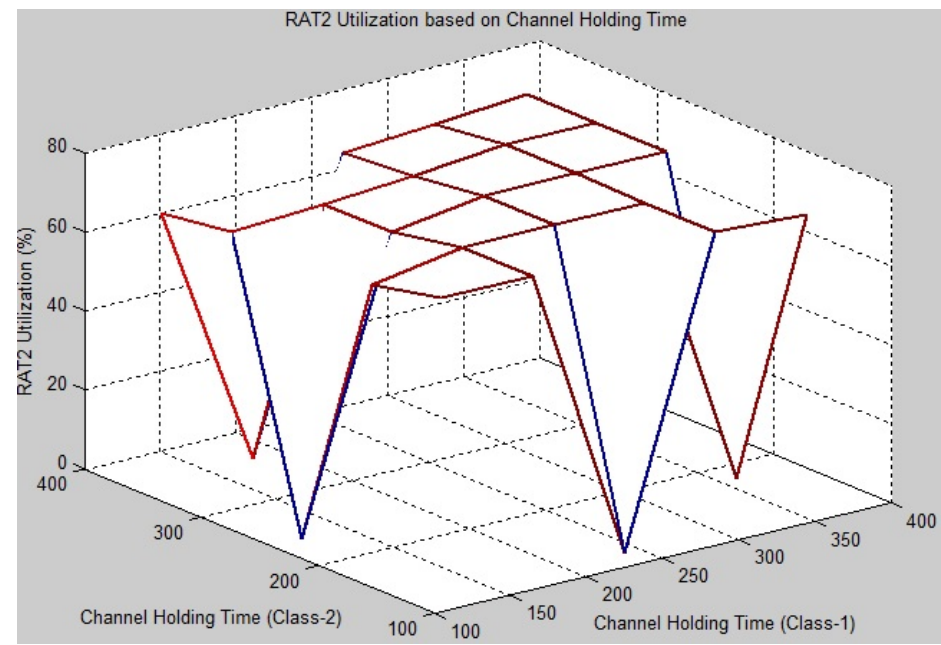

(b)

Figure 4.11: Scenario IV - RAT utilization vs. Channel holding time (a) Case of RAT1 and (b) Case of RAT2

In Fig. 4.12, it can be observed that the optimal cost becomes $0 \%$ when the channel holding time for class- 1 was 180 seconds and 360 seconds. At the same time, it can be observed that when the channel holding time for class-2 is 180 seconds, the optimal cost becomes $0 \%$. 


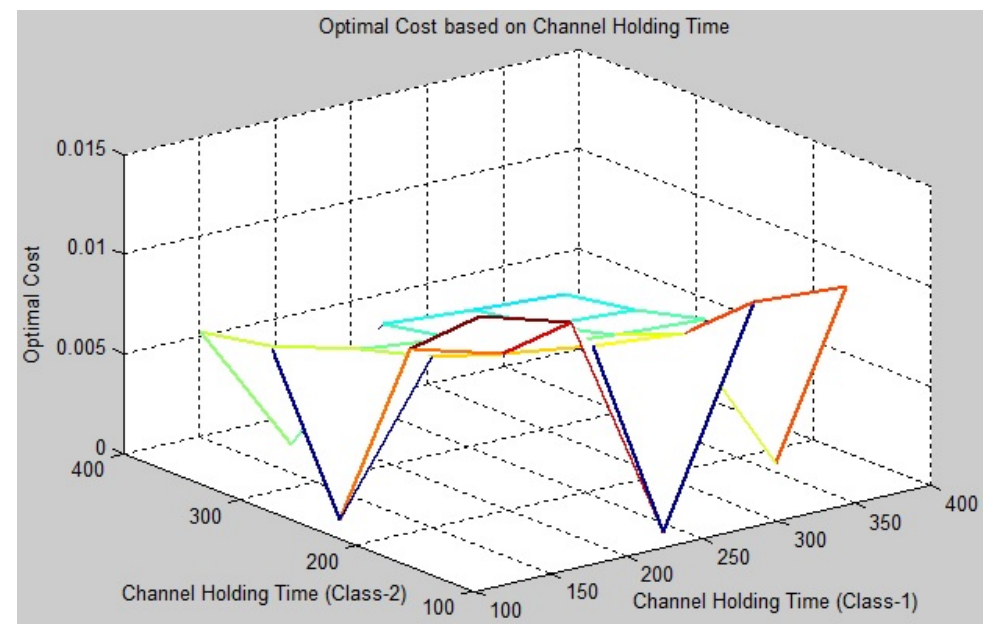

Figure 4.12: Scenario IV - Optimal cost vs. Channel holding time.

\subsection{Analysis of the Optimal Structure of Initial RAT Selection Policy}

In this section, we analyze the behavior of our proposed optimal policy under the system configuration shown in Table 4.6, with focus on determining how the policy allocates the different calls to all existing RATs under different loads (i.e. the number of class- 1 and class- 2 calls in each RAT) in order to achieve optimality. 
Table 4.6: Optimal Policy for the System Configuration

\begin{tabular}{cc|cc|cc}
\hline & \multicolumn{2}{c|}{ Scenarios-I } & \multicolumn{2}{c|}{ Scenarios-II } & \multicolumn{2}{c}{ Scenarios-III } \\
\hline \hline Parameter & Value & Parameter & Value & Parameter & Value \\
\hline$N_{1}$ & 24 channels & $\mu_{1}$ & $1 / 120$ s (voice) & $b_{1}$ & 2 channels \\
$N_{2}$ & 12 channels & $\mu_{2}$ & $1 / 120$ s (voice) & $b_{2}$ & 1 channel \\
$\rho_{1}$ & 5 & $\omega_{1}$ & 0.6 & & \\
$\rho_{2}$ & 3 & $\omega_{2}$ & 0.2 & & \\
& & $\omega_{3}$ & 0.2 & & \\
& & & & & \\
\hline
\end{tabular}

\subsubsection{Analysis of Class-1 Call Accepted by the Optimal JCAC:}

We adopt the following convention (in Tables 4.7 to 4.9 ) to analyze the structure of the optimal policy for class-1 call:

- '-' denotes class-1 call accepted into RAT1.

- '+' denotes class-1 call accepted into RAT2.

- 'a, b' means class-1 call usually accepted into RAT2. But, RAT1 starts taking class-1 call when number of class-1 calls in RAT1 is equal to a or higher and the number of class- 2 calls in RAT1 is equal to $b$ or lower, i.e. $(a, b)$ or $(a+1$, $b-1)$.

- ' $\mathrm{F}$ ' denotes blocking of a call.

In Table 4.7 to Table 4.9 summarize the results obtained in case of class- 1 calls. It can be observed that when the number of class- 2 calls in RAT-2 is 0 , regardless of the RAT1 resource occupancy, the optimal JCAC decides to take class- 1 calls in RAT-2 (+) until RAT-2 resources are fully utilized. 
In Table 4.7, it can be observed that when the number of class-2 calls in RAT-2 is greater than one, and the radio resource occupancy of RAT1 is low (note that RAT1 load is less than 12 out of 24), the optimal JCAC decides to accept class- 1 call in RAT1 (-).

Table 4.7: When RAT1 channel load is less than 12

\begin{tabular}{|c|c|c|c|c|c|c|c|c|c|c|c|c|c|c|}
\hline \multicolumn{15}{|c|}{ No of class- 2 calls in RAT2 } \\
\hline & & 0 & 1 & 2 & 3 & 4 & 5 & 6 & 7 & 8 & 9 & 10 & 11 & 12 \\
\hline & 0 & + & + & - & - & - & - & - & - & - & - & - & - & - \\
\hline$\underset{\Xi}{\leftrightarrows}$ & 1 & + & + & - & - & - & - & - & - & - & - & - & & \\
\hline$\stackrel{0}{\bar{\tau}}$ & 2 & + & + & - & - & - & - & - & - & - & & & & \\
\hline$\vec{\phi}$ & 3 & + & + & - & - & - & - & - & & & & & & \\
\hline$\frac{\pi}{0}$ & 4 & + & + & - & - & - & & & & & & & & \\
\hline$\stackrel{\circ}{Z}$ & 5 & + & + & - & & & & & & & & & & \\
\hline & 6 & - & & & & & & & & & & & & \\
\hline
\end{tabular}

In Table 4.8, it can be observed that when the radio resource occupancy of RAT1 is high, (for example RAT1 load is 16) the optimal JCAC chooses to take class-1 calls in RAT2 (+) till RAT2 resources are fully utilized. However, it is also slower for class-1 calls to go through RAT1 (-). 
Table 4.8: When RAT1 channel load is less than 16

\begin{tabular}{|c|c|c|c|c|c|c|c|c|c|c|c|c|c|c|}
\hline \multicolumn{15}{|c|}{ No of class- 2 calls in RAT2 } \\
\hline & & 0 & 1 & 2 & 3 & 4 & 5 & 6 & 7 & 8 & 9 & 10 & 11 & 12 \\
\hline \multirow{7}{*}{ 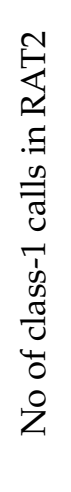 } & 0 & + & + & 11,0 & 10,1 & 9,2 & 8,3 & 7,7 & 6,8 & 5,9 & 4,10 & 3,7 & - & - \\
\hline & 1 & + & 11,0 & 11,0 & 10,1 & 10,1 & 8,3 & 7,7 & 6,8 & 5,9 & - & - & & \\
\hline & 2 & + & 11,0 & 11,0 & 10,1 & 10,1 & 8,3 & 7,7 & - & - & & & & \\
\hline & 3 & + & 11,0 & 11,0 & 10,1 & 10,1 & - & - & & & & & & \\
\hline & 4 & + & 11,0 & 11,0 & - & - & & & & & & & & \\
\hline & 5 & + & 11,0 & - & & & & & & & & & & \\
\hline & 6 & - & & & & & & & & & & & & \\
\hline
\end{tabular}

In Table 4.9, it can be observed that when there is no extra occupancy to accept class-1 call in RAT1, for example, when RAT1 load is 23 to 24 , the optimal JCAC chooses to take class-1 call in RAT2 (+) till RAT2 resources are fully utilized. When there is no more occupancy to accept class-1 call in either RAT1 or RAT2.

Table 4.9: When RAT1 channel load is Grater than 22

\begin{tabular}{|c|c|c|c|c|c|c|c|c|c|c|c|c|c|c|}
\hline \multicolumn{15}{|c|}{ No of class- 2 calls in RAT2 } \\
\hline & & 0 & 1 & 2 & 3 & 4 & 5 & 6 & 7 & 8 & 9 & 10 & 11 & 12 \\
\hline \multirow{7}{*}{ 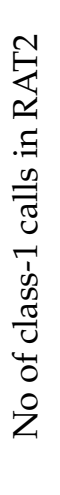 } & 0 & + & + & + & + & + & + & + & + & + & + & + & + & B \\
\hline & 1 & + & + & + & + & + & + & + & + & + & B & B & & \\
\hline & 2 & + & + & + & + & + & + & + & B & B & & & & \\
\hline & 3 & + & + & + & + & + & B & B & & & & & & \\
\hline & 4 & + & + & + & B & B & & & & & & & & \\
\hline & 5 & + & B & B & & & & & & & & & & \\
\hline & 6 & B & & & & & & & & & & & & \\
\hline
\end{tabular}




\section{Chapter 5}

\section{Conclusion}

In the next generation wireless networks, it is expected that different RATs will work in collaboration to provide seamless and ubiquitous access to users. To achieve this objective, a mechanism referred to as Joint Call Admission Control (JCAC) has to be designed with two goals: (1) deciding on the acceptance or rejection of an incoming request from the user; and (2) determining on which of the available RATs the incoming request should be serviced. This thesis has proposed such a method.

More precisely, we have used the framework of Semi-Markov Decision Process (SMDP) to formulate the RAT selection problem in a HetNet composed of two co-located wireless networks (RATs). Our optimal JCAC scheme for initial RAT selection in HetNets is meant to support both real-time and non-real-time services. To meet the JCAC objectives, we have introduced a network cost function that weights three criteria: (a) the blocking cost function, which considers the priority of each service class in each RAT (two service classes are considered); (b) the network 
access cost, which determines a cost associated with accessing a service of a certain type in a given RAT; and (c) the energy consumption cost, which represents the cost of the energy required to operate a base station or an access point. The values that are assigned to these weighted functions can be adjusted according to the mobile network operator's need. In order to compute the JCAC optimal policy, we have utilized the well-known Value Iteration algorithm. It is worth mentioning that our resulting JCAC optimal policy does follow the design trend in next generation wireless networks as well as the expectations that were set by the 3rd Generation Partnership Project (3GPP) group.

To validate our proposed SMDP-based model by simulations, we have considered a HetNet made of two co-located wireless networks (namely representation of GMS and UMTS technologies), under various scenarios and performance metrics. Our simulation results demonstrate the following observation:

(1) Variations in the weights of the blocking cost and the energy consumption cost can greatly impact both the system capacity and the network energy consumption.

(2) When the traffic intensities increase, the blocking probabilities also increase, for a fixed system capacity. However, when the traffic intensities are smaller, the JCAC policy accepts more calls in the less energy consumed RAT, showing the benefit of our design in terms of saving energy while preserving the system performance.

(3) For the scenarios investigated in this thesis, our SMDP model generated 24,842 pairs of state-action for the two co-located wireless networks. Our JRRM 
scheme achieves a good balance between energy-efficient network design and system performance by properly setting up the values of the blocking probability cost function.

(4) Numerical results, supported by an analysis of the structure of the optimal policy, have shown that our proposed optimal JCAC policy selects the best suitable RAT to handle the user's request.

Given the simplicity and robustness of its embedded decision making strategy, our proposed JCAC scheme can be employed in practice by mobile network operators as a feasible and eco-friendly design solution that can successfully tackle the escalation problem of RAT selection in urban areas.

As future works, we believe that our proposed SMDP-based model can be extended to support more sophisticated HetNets architectures (i.e. a HetNet architecture composed of more than two co-located wireless networks and supporting several service classes). Of course, such system will involve a huge number of states and actions that should be deal with. Another challenge is to investigate the problem of inter-RAT handover for such type of architecture. Indeed, from a modeling standpoint, this problem represents a stochastic process in which the state variables can be viewed as handover traffic classes (both vertical and horizontal) and the considered actions are the priority among the traffic classes. In this case, the access network functions (such as functions for discovery and selection) are based on the user's subscription, which in turn, contain some key information such as the type of allowable services, the type of RATs, to name a few, etc. 


\section{Bibliography}

[1] M. López-Benítez and J. Gozalvez, “Common radio resource management algorithms for multimedia heterogeneous wireless networks," IEEE Transactions on Mobile Computing, vol. 10, no. 9, pp. 1201-1213, 2011.

[2] M. Alkhawlani, F. Al-Akwaa, and A. Mohsen, "Intelligent joint admission control for next generation wireless networks," International Journal of Advanced Computer Science and Applications, vol. 3, no. 4, pp. 50-55, 2012.

[3] J. Sachs and M. Olsson, "Access network discovery and selection in the evolved 3gpp multi-access system architecture," European Transactions on Telecommunications, vol. 21, no. 6, pp. 544-557, 2010.

[4] O. Falowo and H. Chan, "Joint call admission control algorithms: Requirements, approaches, and design considerations," Computer Communications, vol. 31, no. 6, pp. 1200-1217, 2008.

[5] G. H. Carvalho, I. Woungang, M. S. Obaidat, A. Anpalagan, and M. M. Rahman, "A joint call admission control-based approach for initial rat selection in hetnets," in Proc. of the IEEE International Conference on Computer, Informa- 
tion and Telecommunication Systems (CITS 2013) Piraeus-Athens, Greece, May 7-8, 2013, pp. 1-5.

[6] M. Alkhawlani and A. Ayesh, "Access network selection based on fuzzy logic and genetic algorithms," Advances in Artificial Intelligence, vol. 8, no. 1, 2008.

[7] H. Tijms, A first course in stochastic models. Wiley, ISBN-13: $978-0-12-$ $398650-4,2003$.

[8] R. Bendlin, V. Chandrasekhar, R. Chen, A. Ekpenyong, and E. Onggosanusi, "From homogeneous to heterogeneous networks: A 3gpp long term evolution rel. 8/9 case study," in Proc. of IEEE 45th Annual Conference on Information Sciences and Systems (CISS), Baltimore, USA, March 23-25, 2011, pp. 1-5.

[9] J. Peng, H. Xian, X. Zhang, and Z. Li, “Context-aware vertical handoff decision scheme in heterogeneous wireless networks," in Proc. of IEEE 10th International Conference on Trust, Security and Privacy in Computing and Communications (TrustCom), Changsha, China, November 16-18, 2011, pp. 1375-1380.

[10] X. Wu, B. Murherjee, and D. Ghosal, "Hierarchical architectures in the thirdgeneration cellular network," IEEE on Wireless Communications,, vol. 11, no. 3, pp. 62-71, 2004.

[11] X. Gelabert, J. Pérez-Romero, O. Sallent, and R. Agustí, “A markovian approach to radio access technology selection in heterogeneous multiaccess/multiservice wireless networks," IEEE Trans. on Mobile Computing, vol. 7, no. 10, pp. 1257-1270, 2008. 
[12] A. Guerrero-Ibáñez, J. Contreras-Castillo, A. Barba, and A. Reyes, "A qosbased dynamic pricing approach for services provisioning in heterogeneous wireless access networks," Pervasive and Mobile Computing, vol. 7, no. 5, pp. 569-583, 2011.

[13] Z. Shi and Q. Zhu, "Radio resource management scheme for heterogeneous wireless networks based on access proportion optimization," Journal of Communications and Networks, vol. 15, no. 5, pp. 527-537, 2013.

[14] M. C. Ertürk, I. Güvenç, S. Mukherjee, and H. Arslan, “Fair and qos-oriented resource management in heterogeneous networks," EURASIP Journal on Wireless Communications and Networking, vol. 2013, no. 1, pp. 1-14, 2013.

[15] A. Liu, V. K. Lau, L. Ruan, J. Chen, and D. Xiao, "Hierarchical radio resource optimization for heterogeneous networks with enhanced inter-cell interference coordination (eicic)," IEEE Transactions on signal processing, vol. 62, no. 7, Apr. 2014.

[16] M. Rafie, "Multi-technology synergies: Multirat small cells radio resource management." [Online]. Available: http://www.argela.com/wp-content/uploads/2013/11/ Multi-Technology-Synergies-Multi-RAT-Small-Cells-RRM.pdf

[17] H. Saraee, S. M. Matinkhah, and S. Khorsandi, "Cooperative joint radio resource management in wireless heterogeneous networks," in Proc. of IEEE International Symposium on Computer Networks and Distributed Systems (CNDS 2011), Feb 23 and 24, Tehran, Iran 2011, pp. 111-115. 
[18] K. Piamrat, A. Ksentini, J. Bonnin, and C. Viho, “Radio resource management in emerging heterogeneous wireless networks," Computer Communications, vol. 34, no. 9, pp. 1066-1076, 2011.

[19] O. Falowo and H. Anthony Chan, "Dynamic rat selection for multiple calls in heterogeneous wireless networks using group decision-making technique," Computer Networks, vol. 56, pp. 1390-1401, 2012.

[20] K. Haldar, C. Ghosh, and D. Agrawal, “Dynamic spectrum access and network selection in heterogeneous cognitive wireless networks," Pervasive and Mobile Computing, vol. 9, pp. 484-497, Aug. 2013.

[21] J. Pérez-Romero, O. Sallent, and R. Agustí, "Policy-based initial rat selection algorithms in heterogeneous networks," in Proc. of the 7th International Conference on Mobile and Wireless Communications Network (MWCN), Marrakesh, Morocco, September 19-21, 2005.

[22] O. E. Falowo and H. A. Chan, "Adaptive bandwidth management and joint call admission control to enhance system utilization and qos in heterogeneous wireless networks," EURASIP Journal on Wireless Communications and Networking, 2007, 2007:034378 doi:10.1155/2007/34378.

[23] S. Jin, W. Xuanli, and S. Xuejun, "Load balancing algorithm with multi-service in heterogeneous wireless networks," in Proc. of 6th IEEE International Conference on Communications and Networking (ICST 2011) Harbin, China, August 17-19, 2011, pp. 703-707. 
[24] M. Lucas-Estañ, J. Gozalvez, and J. Sanchez-Soriano, “Integer linear programming optimization of joint rrm policies for heterogeneous wireless systems," Computer Networks, vol. 56, no. 1, pp. 112-126, 2012.

[25] M. Porjazoski and B. Popovski, "Radio access technology selection algorithm for heterogeneous wireless networks based on service type, user mobility and network load," in proc. of 10th International Conference on Telecommunication in Modern Satellite Cable and Broadcasting Services (TELSIKS),Nis, Serbia, October 5-8, 2011, vol. 2, pp. 475-478.

[26] L. Mohamed, C. Leghris, and A. Abdellah, "A survey and comparison study on weighting algorithms for access network selection," in proc. of IEEE 9th Annual Conference on Wireless On-demand Network Systems and Services (WONS), Courmayeur, Italy, January 9-11, 2012, pp. 35-38.

[27] P. Si, H. Ji, and F. Yu, “Optimal network selection in heterogeneous wireless multimedia networks," Wireless Networks, vol. 16, no. 5, pp. 1277-1288, 2010.

[28] P. Kosmides, A. Rouskas, and M. Anagnostou, “Utility-based $\{R A T\}$ selection optimization in heterogeneous wireless networks," Pervasive and Mobile Computing. [Online]. Available: http://www.sciencedirect.com/science/article/ pii/S1574119213000588

[29] I. Modeas, A. Kaloxylos, and L. Merakos, “Uds: A distributed rat selection mechanism for heterogeneous wireless networks," Wireless Personal Communications, vol. 74, no. 1, pp. 101-132, 2014. 
[30] A. A. Sabbagh, R. Braun, and M. Abolhasan, "A comprehensive survey on rat selection algorithms for heterogeneous networks," World Academy of Science, Engineering and Technology (WASET), no. 73, pp. 141-145, 2011. 